\title{
Does Antenatal Fear of Childbirth Predict Postnatal Fear of Childbirth? A Study of Japanese Women
}

\author{
Mizuki Takegata ${ }^{*}$, Megumi Haruna', Masayo Matsuzaki', Mie Shiraishi' ${ }^{1}$, \\ Tadaharu Okano², Elisabeth Severinsson ${ }^{3}$ \\ ${ }^{1}$ Department of Midwifery and Women's Health, Division of Health Sciences \& Nursing, Graduate School of \\ Medicine, The University of Tokyo, Tokyo, Japan \\ ${ }^{2}$ Center of Physical and Mental Health, Mie University, Tsu City, Japan \\ ${ }^{3}$ Centre for Women's, Family \& Child Health, Department of Nursing Science, Faculty of Health Sciences, \\ Buskerud \& Vestfold University College, Kongsberg, Norway \\ Email: *mharuna-tky@umin.ac.jp
}

Received 28 January 2015; accepted 13 February 2015; published 17 February 2015

Copyright (C) 2015 by authors and Scientific Research Publishing Inc.

This work is licensed under the Creative Commons Attribution International License (CC BY).

http://creativecommons.org/licenses/by/4.0/

(c) (i) Open Access

\begin{abstract}
The study aimed to determine whether antenatal fear of childbirth (expectation) could predict postnatal fear of childbirth (experience) by taking account of other antenatal psychological variables (anxiety and depression) as well as birth outcomes in Japanese women. A longitudinal observational study was conducted at a clinic in Tokyo, Japan, in 2011. Self-report questionnaires were distributed to 240 Japanese women at 37 gestational weeks (Time 1) and on the second day after delivery (Time 2). Regression analyses by means of structural equation modelling were conducted in both the primiparous and the multiparous group. The models exhibited good fit (chisquare value/degree of freedom $=1.10-1.62$, comparative fit index $=0.92-0.99$ and root mean square error of approximation $=0.03-0.07$ ). Antenatal fear of childbirth was the most predictive variable of postnatal fear of childbirth in both the primiparous $(\beta=0.58, p=0.002)$ and the multiparous group $(\beta=0.62, p<0.001)$. In conclusion, antenatal fear of childbirth was a significant predictor of postnatal fear of childbirth when other antenatal psychological variables and birth outcomes were taken into account. Pregnant women who are strongly afraid of childbirth need special attention before and after delivery.
\end{abstract}

\section{Keywords}

Antenatal Fear of Childbirth, Japanese Women, Postnatal Fear of Childbirth, Relationship

\footnotetext{
${ }^{*}$ Corresponding author.
}

How to cite this paper: Takegata, M., Haruna, M., Matsuzaki, M., Shiraishi, M., Okano, T. and Severinsson, E. (2015) Does Antenatal Fear of Childbirth Predict Postnatal Fear of Childbirth? A Study of Japanese Women. Open Journal of Nursing, 5, 144-152. http://dx.doi.org/10.4236/ojn.2015.52017 


\section{Introduction}

Childbirth can be both a challenging and a significant life event. Women may have positive and negative feelings towards the birth. A negative feeling can include fear of childbirth, which involves the child's health, pain, surgical interventions, a difficult course of labour, loss of control and isolation [1]-[3]. Antenatal fear of childbirth is defined as a negative experience of childbirth during pregnancy, while postnatal fear of childbirth is a negative experiences of childbirth brought by fear [4]. Women are afraid of risks to their own health such as haemorrhage, Ceasearean section and forceps/vacuum delivery as well as to that of the child such as being injured and distressed, which are due to the unpredictability of childbirth [3]. In addition, pregnant women are worried about losing control and being isolated during labour, which may lower their confidence and lead to a sense of failure [3].

In the UK and Nordic countries, severe antenatal fear of childbirth has been found in $11 \%$ - $15 \%$ of pregnant women [5] [6] some of whom report strong anxiety, fatigue, and sleep problems [7]. Such women are more likely to consider their childbirth experiences frightening [8] [9]. The negative experience brought about by fear (postnatal fear of childbirth) is associated with traumatic stress symptoms during the postpartum period [10]. Therefore, it is generally recognized that antenatal fear of childbirth can lead to adverse effects on the subjective birth experience and maternal mental state during the postpartum period. In order to reduce the risk of traumatic stress during this period, it is necessary to alleviate the fear of pregnant women who have a strong fear of childbirth before instead of after delivery.

Despite the above recommendation, there have been methodological limitations in previous studies aimed at demonstrating that antenatal fear of childbirth is a predictor of postnatal fear of childbirth. The Wijma Delivery Expectancy/Experience Questionnaire (W-DEQ), which assess fear of childbirth, were compared before and after delivery by means of analyse of variance (ANOVA) among Swedish and Australian samples [8] [9]. These authors found that pregnant women in the high antenatal fear of childbirth group also had high postnatal fear of childbirth scores $(p=0.61)$. However, postnatal fear of childbirth may be associated with other psychosocial variables during pregnancy. First, women who reported strong general anxiety and depression during pregnancy tended to consider childbirth as a negative event [10] [11]. Second, invasive birth outcomes including emergency Caesarean section (emCS), instrumental delivery and prolonged labour have been found to increase negative feelings [12]. Therefore, it is necessary to consider other antenatal psychological aspects in addition to birth outcome.

Furthermore, there is little evidence of fear of childbirth among Japanese women. Antenatal and postnatal fear of childbirth might be influenced by the Japanese cultural context regarding childbirth. For example, the use of epidural anaesthesia is very rare in Japanese clinics and hospitals [13]. According to a previous survey among non-pregnant Japanese women, about $80 \%$ of them would prefer to deliver without anaesthesia [14]. Although several reasons can be considered, one is that Japanese women consider it valuable to experience and overcome labour pain before becoming a mother [14]. Thus the expectation and experience of childbirth among Japanese women may be qualitatively different to those in other countries.

\section{Aim}

The present study aimed to determine whether antenatal fear of childbirth can predict postnatal fear of childbirth by taking account of other antenatal psychological variables (anxiety and depression) as well as birth outcomes in Japanese women.

\section{Material and Methods}

\subsection{Participants and Procedure}

Healthy and Japanese women at 37 gestational weeks were invited to participate in the study by a researcher (M.T.) at an obstetric clinic in Tokyo, between July and November 2011. Women who were less than 20 years old, illiterate in Japanese, hospitalized because of major pregnancy complications or who suffered from serious mental illness were excluded. Women who had planned Caesarean sections were not recruited because the expectancy and experience related to childbirth would be totally different from those undergoing vaginal delivery. However, women who underwent emergency Ceasarean sections (emCS) after the onset of regular labour were included, because they experience the delivery process in a somewhat similar way to women who deliver vagi- 
nally. Of the 246 women recruited, 240 (97.5\%) agreed to participate. They were asked to complete the first questionnaire at 37 gestational weeks (Time 1) and the second questionnaire on the second day after delivery (Time 2).

\subsection{Observational Data at 37 Gestational Weeks (Time 1)}

Data on age, the number of childbirth, education, lifetime prevalence of mental illness, complexity of the pregnancy (pregnancy-induced hypertension, experience of threatened premature labour and placenta previa) and anomalies in the development of the foetus (intrauterine growth restriction and foetalmal formation) were obtained from medical records.

Antenatal fear of childbirth (expectation) was measured by the Japanese W-DEQ (JW-DEQ) version A [4] [15]. Women were asked to answer how they imagine at the moment their delivery will be. The scale consists of 33 items with six-point response alternatives per item, ranging from "not at all” (0) to "extremely" (5); the minimum score is 0 and the maximum 165. A higher score (over 85) indicates severe fear of childbirth and a score of over 100 is considered a phobia; thus, the scale can measure individual degrees of fear [4] and compare primiparous and multiparous women [4]. The JW-DEQ version A identified four factors; Fear, Lack of positive anticipation, Isolation and Riskiness [15], which are consistent with the English and Swedish versions [8] [16] [17]. The Cronbach's alpha value was 0.95 [15].

General symptoms of anxiety and depression during pregnancy were measured by means of the Hospital Anxiety and Depression Scale (HADS). The original scale, developed by Zigmond and Snaith [18] was translated into Japanese by Kitamura et al. [19].

It consists of 14 items with two dimensions (Anxiety: seven items, and Depression: seven items) rated on a four-point scale, ranging from "strongly disagree” (1) to "strongly agree” (4). A higher score indicates a more intense level of general anxiety and depression. In the present study, the Cronbach's alpha of the "Anxiety” subscale was 0.73 and that of the "Depression" subscale 0.60 .

\subsection{Observational Data on the Second Day after Delivery (Time 2)}

Birth outcomes included emCS, vacuum delivery, induced delivery and duration of labour (hours). EmCS was defined as a Caesarean section performed after the onset of regular labour because the experience of childbirth differs between women who only have a planned CS and those who undergo both labour and an emCS.

Postnatal fear of childbirth (experience) was measured with the JW-DEQ version B [4]. Women were asked to answer how they think at the moment their delivery was. The translated JW-DEQ version B also identifies four dimensions consistent with the JW-DEQ version A. The Cronbach's alpha coefficient was 0.94.

\subsection{Statistical Analysis}

Descriptive statistics were summarised as frequency distributions for categorical data and means and standard deviations for continuous data. For descriptive purposes, univariate statistics were obtained for the demographic characteristics by parity. Demographic data such as age and parity, the total HADS score, the total scores of the JW-DEQ versions A and B, in addition to birth outcomes such as duration of labour, emCS, vacuum and induced delivery, were correlated in order to examine their statistical associations (two-tailed statistical significance $p<$ 0.05).

We employed structural equation modelling (SEM) rather than regression analysis because this statistical method is a powerful tool for identifying a hypothesised model of complex causal relationship patterns among variables based on observational data [20]. SEM makes it possible to confirm whether the hypothesised model is applicable to observational data and has the potential to differentiate between observed and latent variables, leading to conclusions that are more valid on the construct level. The two structural regression models differed between the primiparous and the multiparous groups, because women who had an emCS were included in the former [11]. Fear of childbirth factors were considered latent variables in the JW-DEQ versions A and B, which comprised: Fear, Lack of positive anticipation, Isolation and Riskiness [15] [16]. Antenatal fear of childbirth, anxiety, and depression during pregnancy (Time 1), which were determined as exogenous variables, correlated with each other. At the second measurement (Time 2), the exogenous variables duration of labour, vacuum delivery, and induced delivery also correlated with each other. The emCS was included in the primiparous model as an exogenous variable, which correlated with the variables related to birth outcomes. Postnatal fear of child- 
birth determined as an endogenous variable was predicted by antenatal fear of childbirth, anxiety, depression, duration of labour, emCS (only in the primiparous group), vacuum and induced delivery.

These error variables reflect parts of each construct that cannot be explained by the observable variables that form the latent variables. In order to evaluate the fit of the model with the data, chi-square/degree of freedom $\left(\chi^{2} / d f\right)$, comparative fit index (CFI) and root mean square error of approximation (RMSEA) were used as goodness-of-fit indices. According to conventional criteria, $\chi^{2} / d f<3$, CFI $>0.95$, and RMSEA $<0.08$ indicate an acceptable fit while $\chi^{2} / d f<2$, CFI $>0.97$, and RMSEA $<0.05$ indicate a good fit [21]. All statistical analyses were conducted using the Statistical Package for the Social Sciences (SPSS) version 20.0 and Analysis of Moment Structures (Amos) 20.0.

\subsection{Ethical Approval}

Ethical approval for this study was obtained from the Ethics Committee of the University of Tokyo (No. 3417, 2011). All participants were informed about the study, including the fact that anonymity and confidentiality were assured and that they could withdraw at any time by means of a written request.

\section{Results}

Seven (2.9\%) of the 240 participants dropped out on the second day after delivery (Time 2) because they were too exhausted to complete the questionnaire. The response of another participant (which was more than $60 \%$ incomplete) was excluded from the analysis in order to maintain the quality of the data. Eight questionnaires (3.3\%) that had one missing item were completed by the addition of the mean score of the items in question. There were no statistical differences in the demographic data and scores of the psychometrical measures between participants with and without one or more missing items. In this study, seven women (3.0\%) had an emCS, all of whom were primiparas. Therefore, tha data of 232 out of 240 participants' data (96.6\%) were analysed. Table 1 presents the characteristics of the sample.

The mean score (SD) of the JW-DEQ version A was 51.90 (19.47) for primiparas and 46.32 (23.98) for multiparas $(p=0.03)$. The mean score (SD) of the JW-DEQ version B was $64.25(27.42)$ for primiparas and 42.30 (25.75) for multiparas $(p<0.001)$. Four pregnant women in each group (primiparas: $3.2 \%$, multiparas: $3.2 \%)$ had severe fear of childbirth indicated by the JW-DEQ version A scores above 85 . The average duration (SD) of labour was 8.25 (5.09) hours in primiparas and 4.48 (3.00) in multiparas $(p<0.001)$. Eleven primiparas $(9.4 \%)$ and four multiparas $(3.4 \%)$ had vacuum delivery $(p=0.10)$, while 47 primiparas $(40.5 \%)$ and 11 multiparas $(9.4 \%)$ had an induced delivery $(p<0.001)$.

Table 2 presents the correlations of variables. The total scores of the JW-DEQ version B had significant correlations with the factors in the JW-DEQ version A $(r=0.56-0.65, p<0.001)$ and in the HADS subscales (Anxiety: $r=0.42-0.43, p<0.001$, Depression: $r=0.30-0.41, p=0.01$ ). In the primiparous group, the JW-DEQ version B had a significant correlation with vacuum delivery $(r=0.33, p<0.001)$. In the multiparous group, the JW-DEQ version B had a significant correlation with duration of labour ( $r=0.22, p=0.01)$.

Figure 1 and Figure 2 depict the results of the SEM (primiparous group: $\chi^{2} / d f=1.62, p=0.001$, CFI = 0.92, RMSEA $=0.07$, multiparous group: $\chi^{2} / d f=1.10, p=0.28$, CFI $=0.99$, RMSEA $=0.03$ ).

In the result of the SEM regressions analysis, antenatal fear of childbirth $(\beta=0.58, p=0.002)$ and vacuum delivery $(\beta=0.24, p=0.008)$ had a statistical association with postnatal fear of childbirth in the primiparous group. In the multiparous group, antenatal fear of childbirth $(\beta=0.62, p<0.001)$ and duration of labour $(\beta=$ $0.22, p=0.002$ ) had a statistical association with postnatal fear of childbirth. General anxiety and depression during pregnancy did not predict postnatal fear of childbirth, whereas these variables and antenatal fear of childbirth had significant correlations with each other $(r=0.31-0.74, p<0.01)$ in both groups.

In Figure 1 and Figure 2, observational points were at 37 gestational weeks (Time 1) and two days after delivery (Time 2). Fear of childbirth was measured using each factor of the JW-DEQ version A (Time 1) and version B (Time 2). Statistically significant paths are in bold with a standardised path estimate. Non significant paths are with no path estimate shown. e stand for error variable.

\section{Discussion}

The present study demonstrated that antenatal fear of childbirth (expectation) was a significant predictor of 
Table 1. Participants’ characteristics.

\begin{tabular}{|c|c|c|c|c|c|c|c|c|}
\hline & & & & aras & & aras & & \\
\hline & & & & & & & $p$-valu & \\
\hline & Mean \pm & or n (\%) & Mean \pm & or n (\%) & Mean \pm & or n (\%) & & \\
\hline Age & 33.69 & \pm 4.00 & 32.01 & \pm 4.10 & 33.94 & \pm 3.75 & $<0.001$ & a \\
\hline Education High school & 17 & (7.3) & 6 & $(5.1)$ & 11 & $(9.4)$ & 0.653 & $\mathrm{~b}$ \\
\hline College & 76 & $(32.7)$ & 41 & (35.3) & 35 & (30.1) & & \\
\hline University & 139 & $(60.0)$ & 69 & (59.6) & 70 & $(60.3)$ & & \\
\hline Planning to deliver with family & 225 & $(96.9)$ & 112 & $(96.5)$ & 113 & $(97.4)$ & 0.512 & b \\
\hline History of psychiatric disorders & 6 & $(2.5)$ & 4 & $(3.4)$ & 2 & $(1.7)$ & 0.252 & $\mathrm{~b}$ \\
\hline Complexity of pregnancy & 37 & $(15.9)$ & 20 & $(17.2)$ & 17 & $(14.6)$ & 0.375 & $\mathrm{~b}$ \\
\hline Anomalies in development of foetus & 7 & (3.0) & 4 & (3.4) & 3 & $(2.5)$ & 0.363 & b \\
\hline Birth outcomes & & & & & & & & \\
\hline Duration of labour (h) & 6.37 & \pm 4.57 & 8.25 & \pm 5.09 & 4.48 & \pm 3.00 & $<0.001$ & a \\
\hline Emergency Caesarean section & 7 & $(3.0)$ & 7 & $(6.0)$ & 0 & (0) & 0.01 & $\mathrm{~b}$ \\
\hline Vacuum delivery & 15 & (6.4) & 11 & (9.4) & 4 & (3.4) & 0.100 & $\mathrm{~b}$ \\
\hline Induced delivery & 58 & $(25.0)$ & 47 & $(40.5)$ & 11 & (9.4) & $<0.001$ & $\mathrm{~b}$ \\
\hline Measures & & & & & & & & \\
\hline 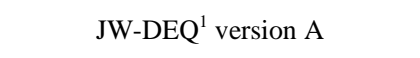 & 49.11 & \pm 21.97 & 51.90 & \pm 19.47 & 46.32 & \pm 23.98 & 0.031 & a \\
\hline HADS $^{2}$ anxiety & 4.97 & \pm 3.61 & 4.82 & \pm 3.78 & 5.12 & \pm 3.45 & 0.522 & a \\
\hline $\mathrm{HADS}^{2}$ depression & 2.94 & \pm 2.08 & 2.89 & \pm 2.13 & 2.99 & \pm 2.05 & 0.731 & a \\
\hline $\mathrm{JW}^{-\mathrm{DEQ}^{1}}$ version B & 53.28 & \pm 28.73 & 64.25 & \pm 27.42 & 42.30 & \pm 25.75 & $<0.001$ & $\mathrm{a}$ \\
\hline
\end{tabular}

${ }^{1}$ The Japanese version of the Wijma Delivery Expectancy/Experience Questionnaire (33 items, scored 0 - 165). Version A: antentatal fear of childbirth, Version B: postnatal fear of childbirth. ${ }^{2}$ The Hospital anxiety and depression scale (Anxiety: 7 items, scored 0 - 21; Depression: 7 items, scored 0 - 21). ${ }^{\mathrm{a}}$ Student's t-test, ${ }^{\mathrm{b}}$ Chi-squared test, Statistical significance: $p<0.05$.

postnatal fear of childbirth (experience) when other psychological variables (general depression and anxiety) during pregnancy and birth outcomes (duration of labour, emCS, vacuum and induced delivery) were taken into account in primiparous and multiparous groups.

The results provide robust supports to previous findings that antenatal fear of childbirth is a predictor of postnatal fear of childbirth [6] [8]. In addition, Garthus-Niegel et al. [11] demonstrated the statistical association between antenatal fear of childbirth and negative birth experience by asking the mothers three ad-hoc questions (the content of subjective birth experience was fear, overall dissatisfaction and dissatisfaction with care) ( $\beta=$ $0.44, p<0.001)$. The statistical association was stronger than that between birth outcomes and negative birth experience $(\beta=0.25, p<0.001)$. Postnatal fear of childbirth can be categorised into one psychological aspect, namely negative birth experience. In our results, antenatal fear of childbirth is a better predictor of postnatal fear of childbirth than birth outcome. Thus, our results were similar to Gathus-Niegel's findings [11], although the measures of birth experience differed in content between this and the previous study [11]. However, we are unable to confirm that antenatal fear of childbirth is a strong predictor of negative birth experience than birth outcome because the majority of women in this study delivered normally. Nevertheless, we suggest that a psychological intervention as well as postnatal support are necessary for pregnant women who have a strong fear of childbirth before delivery. 
Table 2. Statistical correlations $(\mathrm{n}=232)$.

\begin{tabular}{|c|c|c|c|c|c|c|c|c|c|}
\hline & 1 & 2 & 3 & 4 & 5 & 6 & 7 & 8 & 9 \\
\hline \multirow{2}{*}{ 1) Age } & 1 & & & & & & & & \\
\hline & 1 & & & & & & & & \\
\hline \multirow{2}{*}{ 2) $J W W E Q^{1}$ version $A$} & 0.02 & 1 & & & & & & & \\
\hline & 0.09 & 1 & & & & & & & \\
\hline \multirow{2}{*}{ 3) $\mathrm{HADS}^{2}$ anxiety } & 0.03 & $0.60^{* * *}$ & 1 & & & & & & \\
\hline & 0.06 & $0.40^{* * *}$ & 1 & & & & & & \\
\hline \multirow{2}{*}{ 4) $\mathrm{HADS}^{2}$ depression } & 0.07 & $0.46^{* * *}$ & $0.73^{* * *}$ & 1 & & & & & \\
\hline & 0.04 & $0.29^{* *}$ & $0.73^{* * *}$ & 1 & & & & & \\
\hline \multirow{2}{*}{ 5) $\mathrm{JWDEQ}^{1}$ version $\mathrm{B}$} & 0.09 & $0.56^{* * *}$ & $0.43^{* * *}$ & $0.41^{*}$ & 1 & & & & \\
\hline & 0.18 & $0.65^{* * *}$ & $0.42^{* * *}$ & $0.30^{*}$ & 1 & & & & \\
\hline \multirow{2}{*}{$\begin{array}{l}\text { 6) Emergency } \\
\text { Caesarean Section }\end{array}$} & 0.03 & 0.00 & 0.07 & 0.02 & 0.16 & 1 & & & \\
\hline & & & & & & 1 & & & \\
\hline \multirow{2}{*}{ 7) Induced delivery } & -0.02 & 0.13 & $0.21^{*}$ & 0.16 & 0.16 & 0.08 & 1 & & \\
\hline & 0.11 & 0.37 & 0.03 & 0.07 & 0.18 & & 1 & & \\
\hline \multirow{2}{*}{ 8) Vacuum delivery } & $0.26^{* *}$ & 0.13 & $0.25^{* *}$ & $0.29^{*}$ & $0.33^{* * *}$ & 0.16 & $0.21^{*}$ & 1 & \\
\hline & 0.01 & 0.06 & -0.10 & 0.02 & 0.13 & & 0.28 & 1 & \\
\hline \multirow{2}{*}{ 9) Duration of labor } & 0.09 & 0.07 & -0.05 & -0.03 & -0.00 & $-0.22^{*}$ & -0.06 & -0.04 & 1 \\
\hline & -0.04 & 0.00 & 0.03 & 0.02 & $0.22^{*}$ & & 0.07 & 0.00 & 1 \\
\hline
\end{tabular}

${ }^{1}$ The Japanese version of the Wijma Delivery Expectancy/Experience Questionnaire (33 items, scored 0 - 165). Version A: antenatal fear of childbirth, Version B: postnatal fear of childbirth. ${ }^{2}$ The hospital anxiety and depression scale (Anxiety: 7 items, scored 0 - 21; Depression: 7 items, scored 0 - 21). Pearson's correlation, Upper: Primiparas, Lower: Multiparas, Statistical significance: ${ }^{*} p<0.05,{ }^{* *} p<0.01,{ }^{* * *} p<0.001$.

Time1: 37 gestational weeks

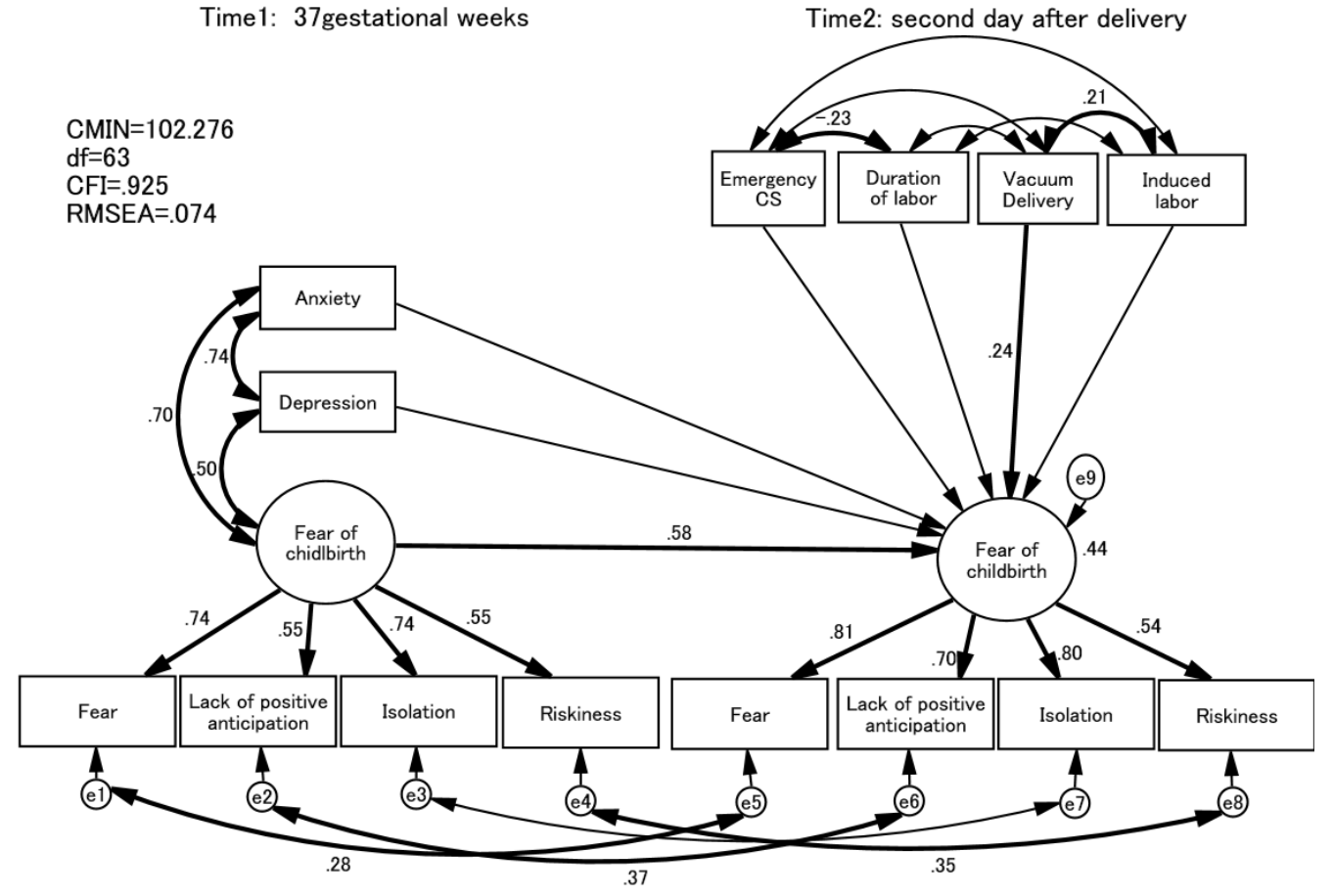

Figure 1. Structural regression model (Primiparas, $n=116$ ). 


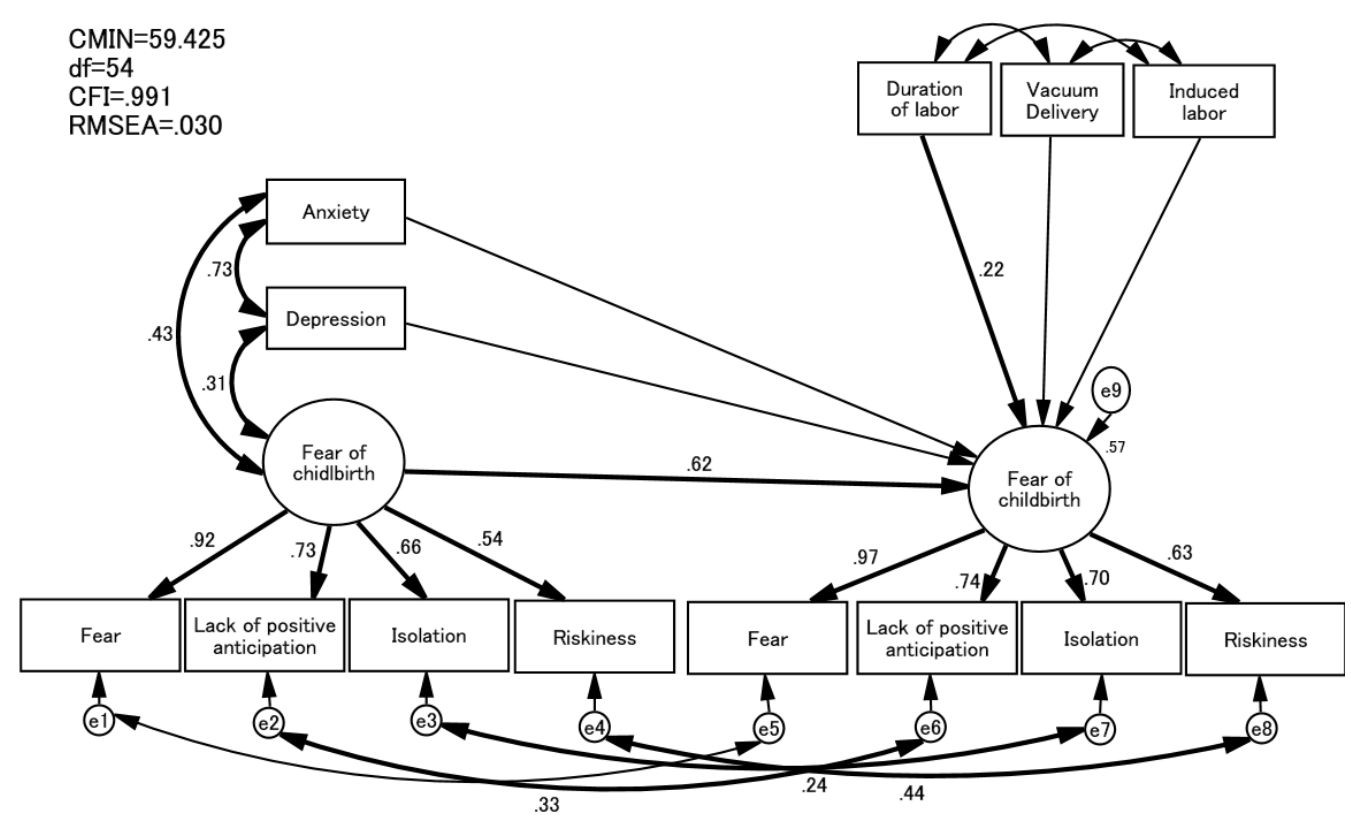

Figure 2. Structural regression model (Multiparas, $\mathrm{n}=116$ ).

With regard to birth outcome, vacuum delivery was the only significant variable associated with postnatal fear of childbirth in the primiparous group. Instrumental delivery is linked to negative birth experience, traumatic stress and emotional distress during the postpartum period because the delivery was an emergency procedure in response to foetal distress or failure to progress [22] [23]. Waldenström [24] stated that primiparous women had more positive expectations of childbirth than multiparous women, but had the disadvantage of complicated labour. Thus, primiparous women who unexpectedly underwent avacuum delivery may lose emotional control and experience intense fear during childbirth. On the other hand, emCS did not have significant associations with postpartum fear of childbirth ( $p=0.09$ ), which can probably be explained by the fact that the number of women who had an emCS was relatively small $(n=7)$ in this study. Another study with a larger number of participants is needed to confirm the results.

In the multiparous group, only duration of labour had a significant association with postnatal fear of childbirth. Most multiparous women expect that the birth will progress faster than their first delivery and do not lose their perception of time during labour as opposed to women in the primiparas group [24]. Therefore, when deliveries take longer than expected, they may feel anxious and fearful. In addition, negative experiences from a previous birth can affect them in different ways.

In the present study, antenatal fear of childbirth was the only psychological variable to predict postnatal fear of childbirth. This is because antenatal fear of childbirth concerns negative feelings specifically related to childbirth [4], while general anxiety and depression encompass negative feelings related not only to childbirth, but also to other negative life events such as minor problems associated with pregnancy and conflicts with family members. Thus, because of their broader concepts, it cannot be assumed that general anxiety and depression during pregnancy are factors that directly contribute to a negative birth experience. Nevertheless, general anxiety and depression during pregnancy were significantly correlated with antenatal fear of childbirth in the present results. Considering the fact that general anxiety and depression interacted with antenatal fear of childbirth, it seems reasonable to assume that pregnant women who have a severe fear of childbirth also exhibit intense general anxiety and depression during pregnancy, which leads to severe postnatal fear of childbirth.

This study has several limitations that need to be addressed. First, this study was conducted in just one setting and therefore the results cannot be generalized. The sample was composed of relatively healthy Japanese women, and their average scores (Mean \pm SD: $49.1 \pm 21.9$ ) of the JW-DEQ A were lower than those of Nordic women $(62.8 \pm 23.3)$ [5]. In addition, the small number of participants who underwent an emCS were only involved the 
primiparas group, while the majority delivered normally. Although the sample is not representative of the total population of pregnant Japanese women, the results initially support the assumption that antenatal fear of childbirth rather than delivery methods is a predictor of postnatal fear of childbirth. A second limitation is that a larger sample with over 300 participants in each group would provide more robust results, although the present sample met the minimum SEM sample size criterion ( $>100$ ) [21]. Thirdly, a negative birth experience contains a variety of concepts such as dissatisfaction, pain and fear [11] [25]. The present study explored postnatal fear of childbirth as one of the psychometric aspects of negative birth experience but other emotional aspects might need to be taken into consideration. A longitudinal follow-up study with a larger sample that examines various psychometric variables is necessary.

\section{Conclusion}

Antenatal fear of childbirth was found to be a strong predictor of postnatal fear of childbirth, when other antenatal psychological variables such as anxiety, depression and birth outcomes were taken into account. Thus, women who are strongly afraid of childbirth require special attention before and after delivery in order to cope with their childbirth experiences in a more positive way.

\section{Acknowledgements}

We would like to thank all participants and nursing staff members for their cooperation. We wish to express our sincere gratitude to Dean Toshinori Kitamura of the Kitamura Institute of Mental Health in Japan for his supervision and support. We also thank Professor Klaas Wijma of Linköping University in Sweden for permission to translate the WDEQ questionnaire to Japanese.

\section{Funding Statement}

This work was supported by a MEXT/JSPS KAKENHI Grant-in-Aid for Challenging Exploratory Research (23660058).

\section{References}

[1] Eriksson, C., Jansson, L. and Hamberg, K. (2006) Women's Experiences of Intense Fear Related to Childbirth Investigated in a Swedish Qualitative Study. Midwifery, 22, 240-248. http://dx.doi.org/10.1016/j.midw.2005.10.002

[2] Geissbuehler, V. and Eberhard, J. (2002) Fear of Childbirth during Pregnancy: A Study of More than 8000 Pregnant Women. Journal of Psychosomatic Obstetrics Gynaecology, 23, 229-235. http://dx.doi.org/10.3109/01674820209074677

[3] Nilsson, C. and Lundgren, I. (2009) Women’s Lived Experience of Fear of Childbirth. Midwifery, 25, 1-9. http://dx.doi.org/10.1016/j.midw.2007.01.017

[4] Wijma, K., Wijma, B. and Zar, M. (1998) Psychometric Aspects of the W-DEQ: A New Questionnaire for the Measurement of Fear of Childbirth. Journal of Psychosomatic Obstetrics Gynaecology, 19, 84-97. http://dx.doi.org/10.3109/01674829809048501

[5] Nieminen, K., Stephansson, O. and Ryding, E.L. (2009) Women’s Fear of Childbirth and Preference for Cesarean Section: A Cross-Sectional Study at Various Stages of Pregnancy in Sweden. Acta Obstetrics Gynecology Scandinavia, 88, 807-813. http://dx.doi.org/10.1080/00016340902998436

[6] Zar, M., Wijma, K. and Wijma, B. (2002) Relations between Anxiety Disorders and Fear of Childbirth during Late Pregnancy. Clinical Psychology Psychotherapy, 9, 122-130. http://dx.doi.org/10.1002/cpp.305

[7] Hall, W.A., Hauck, Y.L., Carty, E.M., Hutton, E.K., Fenwick, J. and Stoll, K. (2009) Childbirth Fear, Anxiety, Fatigue and Sleep Deprivation in Pregnant Women. Journal of Obstetric Gynecologic Neonatal Nursing, 38, 567-576. http://dx.doi.org/10.1111/j.1552-6909.2009.01054.x

[8] Fenwick, J., Gamble, J., Nathan, E., Bayes, S. and Hauck, Y. (2009) Pre- and Postpartum Levels of Childbirth Fear and the Relationship to Birth Outcomes in a Cohort of Australian Women. Journal of Clinical Nursing, 18, 667-677. http://dx.doi.org/10.1111/j.1365-2702.2008.02568.x

[9] Zar, M., Wijma, K. and Wijma, B. (2001) Pre- and Postpartum Fear of Childbirth in Nulliparous and Parous Women. Scandinavian Journal of Behaviour Therapy, 30, 75-84. http://dx.doi.org/10.1080/02845710121310

[10] Verreault, N., Da Costa, D., Marchand, M., Ireland, K., Banack, H., Dritsa, M. and Khalifé M. (2012) PTSD Following 
Childbirth: A Prospective Study of Incidence and Risk Factors in Canadian Women. Journal of Psychosomatic Research, 73, 257-263. http://dx.doi.org/10.1016/j.jpsychores.2012.07.010

[11] Garthus-Niegel, S., Von Soest, T., Vollrath, M.E. and Eberhard-Gran, M. (2013) The Impact of Subjective Birth Experiences on Post-Traumatic Stress Symptoms: A Longitudinal Study. Archives of Women's Mental Health, 16, 1-10. http://dx.doi.org/10.1007/s00737-012-0301-3

[12] Waldenström, U., Hildingsson, I. and Ryding, E.L. (2006) Antenatal Fear of Childbirth and Its Association with Subsequent Caesarean Section and Experience of Childbirth. International Journal of Obstetric Gynaecology, 113, 638646. http://dx.doi.org/10.1111/j.1471-0528.2006.00950.x

[13] Terui, K. (2009) Comparison of the Obstetric Anesthesia Practice between Japan and USA. Masui, 58, 1473-1475. (In Japanese)

[14] Yoshida, K. (2010) Attitudes of Healthcare Practitioners and Consumers toward the "Acceptance” and “Avoidance” of Labor Pains. Boseieisei, 51, 99-109. (In Japanese)

[15] Takegata, M., Haruna, M., Murayama, R., Masayo, M., Okano, T. and Severinsson, E. (2013) Translation and Validation of the Japanese Version of the Wijma Delivery Expectancy/Experience Questionnaire Version A. Nursing and Health Science, 15, 326-332. http://dx.doi.org/10.1111/nhs.12036

[16] Johnson, R. and Slade, P. (2002) Does Fear of Childbirth during Pregnancy Predict Emergency Caesarean Section? BJOG: An International Journal of Obstetrics and Gynaecology, 109, 1213-1221. http://dx.doi.org/10.1046/j.1471-0528.2002.01351.x

[17] Wiklund, I., Edman, G., Ryding, E.L. and Andolf, E. (2008) Expectation and Experiences of Childbirth in Primiparae with Caesarean Section. BJOG: An International Journal of Obstetrics and Gynaecology, 115, 324-331. http://dx.doi.org/10.1111/j.1471-0528.2007.01564.x

[18] Zigmond, A.S. and Snaith, R.P. (1983) The Hospital Anxiety and Depression Scale (HADS). Acta Psychiatrica Scandinavia, 67, 361-370. http://dx.doi.org/10.1111/j.1600-0447.1983.tb09716.x

[19] Kitamura, T. (1993) The Hospital Anxiety and Depression Scale (HADS). Archives of Psychiatric Diagnostics and Clinical Evaluation, 4, 371-372. (In Japanese)

[20] Kline, R.B. (2004) Principles and Practice of Structural Equation Modeling. 2nd Edition, Guilfold Publications Inc., New York.

[21] Byrne, B.M. (2001) Structural Equation Modeling with AMOS: Basic Concepts, Applications, and Programming. Lawrence Erlbaum Associates, Mahwah.

[22] Creedy, D.K., Shochet, I.M. and Horsfall, J. (2000) Childbirth and the Development of Acute Trauma Symptoms: Incidence and Contributing Factors. Birth, 27, 104-111. http://dx.doi.org/10.1046/j.1523-536x.2000.00104.x

[23] Söderquist, J., Wijma, B., Thorbert, G. and Wijma, K. (2009) Risk Factors in Pregnancy for Post-Traumatic Stress and Depression after Childbirth. BJOG: An International Journal of Obstetrics and Gynaecology, 116, 672-680.

[24] Waldenström, U., Borg, I.M., Olsson, B., Sköld, M. and Wall, S. (1996) The Childbirth Experience: A Study of 295 New Mothers. Birth, 31, 17-27.

[25] Goodman, P., Mackey, M.C. and Tavakoli, A.S. (2004) Factors Related to Childbirth Satisfaction. Journal of Advanced Nursing, 46, 212-219. http://dx.doi.org/10.1111/j.1365-2648.2003.02981.x 
Scientific Research Publishing (SCIRP) is one of the largest Open Access journal publishers. It is currently publishing more than 200 open access, online, peer-reviewed journals covering a wide range of academic disciplines. SCIRP serves the worldwide academic communities and contributes to the progress and application of science with its publication.

Other selected journals from SCIRP are listed as below. Submit your manuscript to us via either submit@scirp.org or Online Submission Portal.
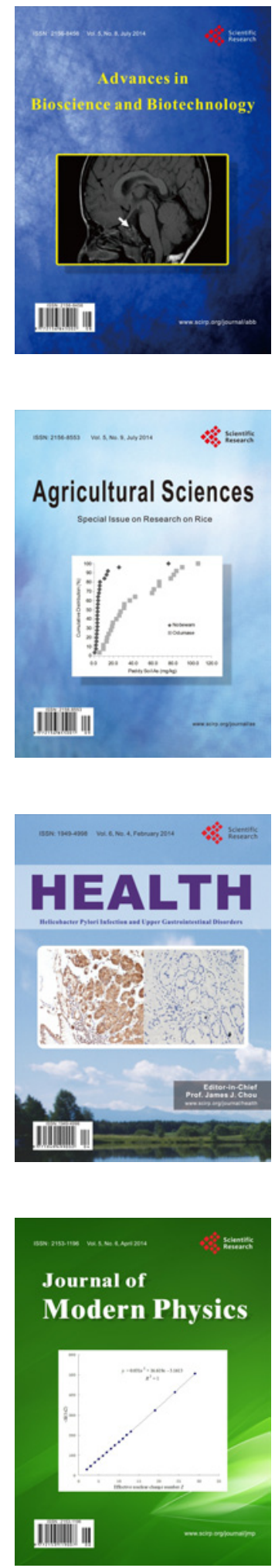
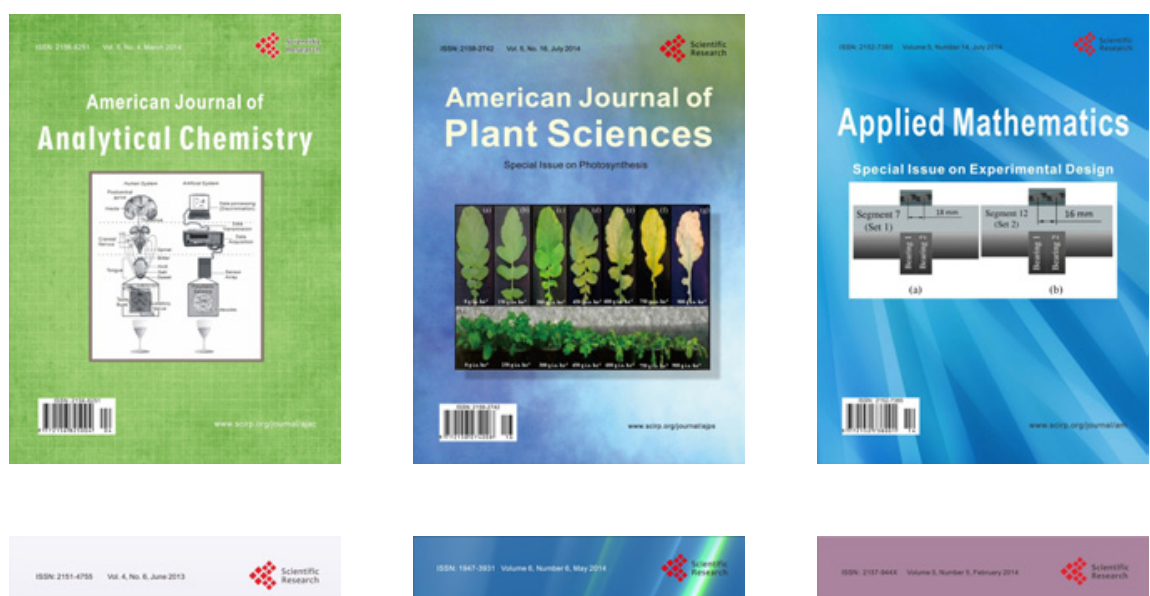

Creative Education
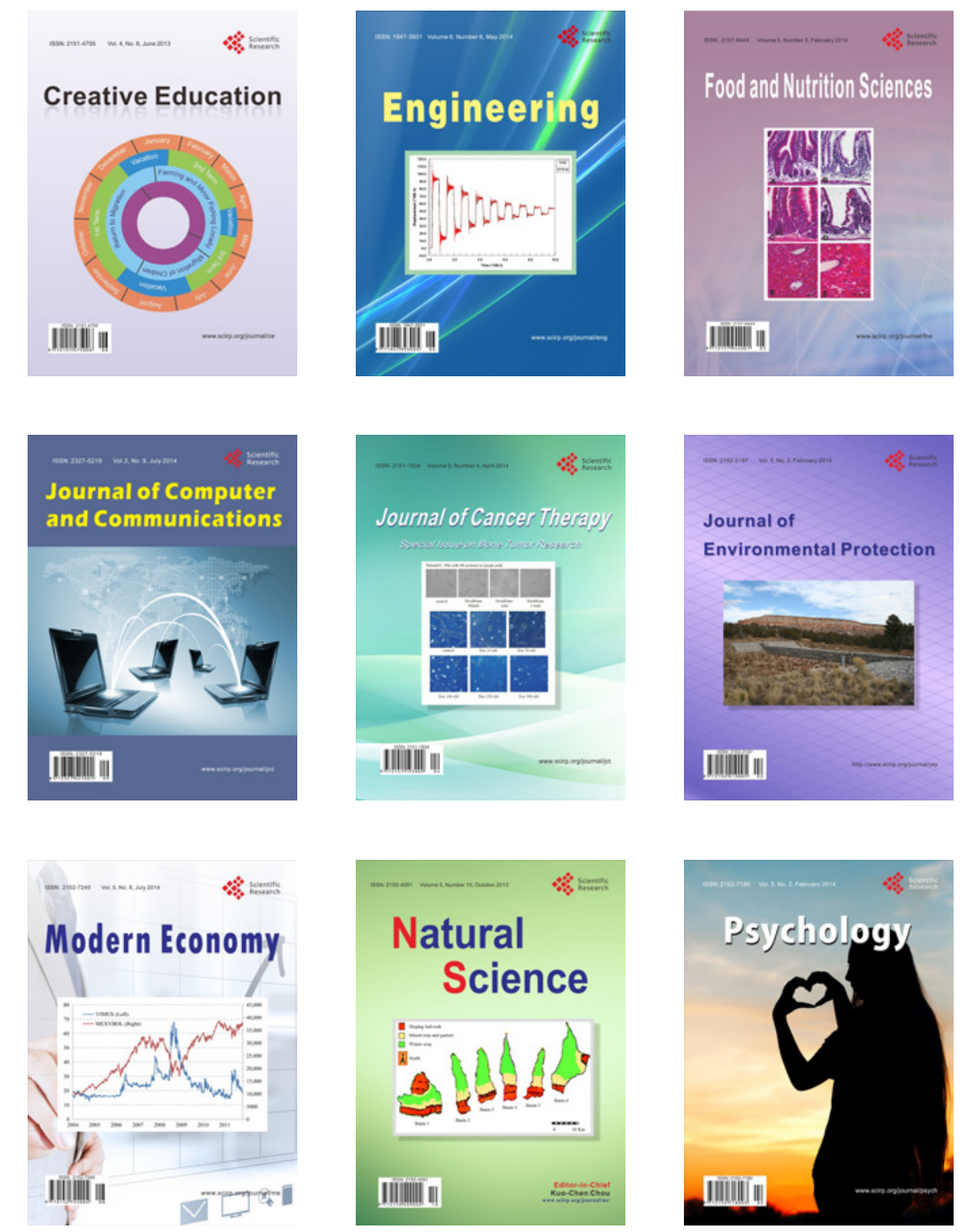